\title{
The impact of irradiation dose on the computed tomography radiographic response of metastatic nodes and clinical outcomes in cervix cancer in a low-resource setting
}

\author{
Matthew Ryan McKeever, Lindsay Hwang', Jennifer Barclay, Yin Xi',April Bailey', Kevin Albuquerque
}

\begin{abstract}
Introduction: The aim of this study is to investigate the relationship between the radiation dose to pelvic and para-aortic lymph nodes, nodal response, and clinical outcomes in a resource-poor setting based on computed tomography (CT) nodal size alone. Materials and Methods: This retrospective study from 2009 to 2015 included 46 cervical cancer patients with I 33 metastatic pelvic and para-aortic lymph nodes definitively treated with chemoradiation and brachytherapy in a public hospital with limited access to positron emission tomography (PET) scans. Hence, short axis of the lymph node on CT scan was used as a measure of metastatic nodal disease, before and following radiation therapy. Inclusion criteria required the pelvic and para-aortic nodes to have the shortest axis diameter on CT scan of $\geq 8 \mathrm{~mm}$ and $\geq 10 \mathrm{~mm}$, respectively. Based on PET resolution, a node that decreased to half of its inclusion cutoff size was considered to have a complete response (CR). Relevant clinical outcomes were documented and correlated with nodal features, nodal radiation doses, and treatment characteristics. Results: After controlling for other predictive factors, increased nodal dose was associated with increased probability of $C R$ per study definition $(P=0.005)$. However, there was no statistically significant association between dose and pelvic/para-aortic, distant and total recurrence $(T R)$, and any recurrence at any location $(P=0.263,0.785, I .00$, respectively). Patients who had no CR nodes had shorter pelvic/para-aortic recurrence-free survival (PPRFS) and TR-free survival (TRFS) than patients who had at least one CR node $(P=0.027$ and 0.046 , respectively). Patients with no $C R$ nodes also had shorter PPRFS than patients who had all nodes completely respond $(P<0.05)$. Conclusions: Using $C T$-based measures, we found that increased nodal dose is associated with an increased probability of CR (as defined) and nodal CR is associated with increased PPRFS and TRFS. We were unable to determine the cutoff dose required for a CR.
\end{abstract}

Key words: Cervical cancer, lymph nodes, oncology, radiation

\section{Introduction}

Locally advanced cervical cancer (LACC) encompasses all cervical cancers that cannot be treated primarily with surgery and is often associated with pelvic and para-aortic lymph node metastasis, which is one of the most significant prognostic factors for recurrence and death. ${ }^{[1]}$ The current standard of care for LACC is a combination of concurrent external beam chemoradiation with high-dose rate (HDR), low- or pulsed-dose rate brachytherapy (BT) boost. However, there have not been definitive recommendations for the optimal dose to pelvic and para-aortic lymph nodes. Radiation techniques enable boosting pelvic and para-aortic lymph nodes, but some researchers have questioned the clinical relevance of giving any boost dose to involved pelvic lymph nodes. ${ }^{[2]}$ However, there is evidence suggesting a dose response for pelvic nodal control. ${ }^{[3]}$ With this in mind, we reviewed the charts of our LACC patients with pelvic and para-aortic lymph nodes to correlate outcomes with radiation dose.

\section{Materials and Methods}

\section{Patients}

This Institutional Review Board approved retrospective study from 2009 to 2015 included 46 LACC patients with a total of 133 metastatic pelvic and para-aortic lymph nodes. To be included in the study, the patients had to have newly diagnosed LACC with involved pelvic or para-aortic lymph nodes and at least one follow-up scan after treatment. For a node to be included in the study, the shortest axis of the node had to be $8 \mathrm{~mm}$ or greater on an axial contrast-enhanced (CE) computed tomography (CT) for pelvic nodes and $10 \mathrm{~mm}$ or greater on axial CT for para-aortic nodes. Positron emission tomography (PET)-CT was available in 39 patients, but due to difficulty/cost in obtaining these scans for

\begin{tabular}{|c|}
\hline Access this article online \\
\hline Quick Response Code: \\
\\
Website: www.sajc.org \\
\hline DOI: 10.4103/2278-330X.208856 \\
\hline
\end{tabular}

Departments of Radiation Oncology and 'Radiology, University of Texas Southwestern Medical School, Dallas, TX, 'Case Western Reserve University School of Medicine, Cleveland, OH, USA

Correspondence to: Dr. Kevin Albuquerque, E-mail: kevin.albuquerque@utsouthwestern.edu all patients, we use CE-CT measurements to classify the nodes as metastatically involved based on the method of Koh et al..$^{[4]}$ PET standardized uptake value (SUV) values were not utilized to define nodal metastases as PET was not available routinely in this resource-poor population. There were a total of 113 involved pelvic node and twenty involved para-aortic nodes. Thirty patients had pelvic nodes only, 14 had pelvic and para-aortic nodes, and two patients had para-aortic nodes only. The median age of the patient was 49 years (range, 22-78 years). The International Federation of Gynecology and Obstetrics stages ranged from IBI to IVA (the single IBI patient was included here because of nodal metastatic disease and was considered to be unresectable). Forty patients had squamous cervical carcinoma, four had cervical adenocarcinoma, one patient had adenosquamos cervical carcinoma, and one had poorly differentiated cervical carcinoma. For concurrent chemotherapy, 43 (93\%) of the patients received cisplatin alone and three $(6 \%)$ received cisplatin with other agents. Median number of cycles was 5. Patient characteristics are shown in Table 1.

\section{Radiation therapy}

All patients received three-dimensional conformal whole pelvic \pm extended field (for para-aortic nodes) radiation therapy to the nodal regions and the cervical primary to a dose of 45 Gy in 25 fractions. CT-size defined involved lymph nodes received a supplemental boost dose. Most of the patients received CT-based HDR BT dosed to point A. For each BT fraction, a plan was created that was optimized to point A dose. Manual optimization was then performed to adapt the dose to the CT-based cervix target and organs at risk.

This is an open access article distributed under the terms of the Creative Commons Attribution-NonCommercial-ShareAlike 3.0 License, which allows others to remix, tweak, and build upon the work non-commercially, as long as the author is credited and the new creations are licensed under the identical terms.

For reprints contact: reprints@ medknow.com

How to cite this article: McKeever MR, Hwang L, Barclay J, Xi Y, Bailey A, Albuquerque $\mathrm{K}$. The impact of irradiation dose on the computed tomography radiographic response of metastatic nodes and clinical outcomes in cervix cancer in a low-resource settingLetter to the Editor. South Asian J Cancer 2017;6:54-8. 
For external beam radiation, all of the patients' treatments were planned using Pinnacle Treatment Planning System Version 9.4 (Philips, Amsterdam, The Netherlands). The lymph nodes were mapped to different regions and measured on CT scans under the supervision of a radiologist. The treatment plan for each patient was then uploaded into Pinnacle. The lymph nodes were contoured, and the dose volume histogram was used to find the mean dose from external beam for each node. The median external beam radiation dose of the 133 lymph nodes was 51.4 Gy (range, 28.5-64.2 Gy).

\section{Follow-up}

As part of routine follow-up, all patients had an initial follow-up CT and $69 \%, 39 \%$, and $24 \%$ of patents had a second, third, and fourth scans, respectively. The median time from the end of treatment to the CT scan was 140, 189, 265, and 272 days for the first, second, third, and fourth follow-up scans, respectively. At each follow-up scan, the node's short axis was measured and the change in size of the node was calculated. The patients were also followed clinically and any failure or recurrence was recorded. The median follow-up time from the end of the treatment was 15 months. A node was considered to have a complete response (CR) if its shortest axis decreased to a size that was half of the inclusion cutoff size ( $4 \mathrm{~mm}$ for pelvic nodes and $5 \mathrm{~mm}$ for para-aortic nodes). Our CR cutoff size was chosen to correspond to the size at which a node would no longer be visible on PET scan. ${ }^{[5]}$ Due to the inherent resolution limitations of PET (with or without $\mathrm{CT}$ ), tumors with a short axis $<5 \mathrm{~mm}$ would not be visible on a PET; ${ }^{[5]}$ hence, this was chosen as the study definition cutoff for nodal CR.

\section{Statistical analysis}

The generalized estimating equation model was used to test the difference in mean external beam dose and mean initial size

Table 1: Patient characteristics

\begin{tabular}{|c|c|c|}
\hline Patient Characteristics & $n$ & $\%$ \\
\hline \multicolumn{3}{|l|}{ Age (years) } \\
\hline $21-30$ & 8 & 17 \\
\hline $31-40$ & 4 & 9 \\
\hline $41-50$ & 16 & 35 \\
\hline $51-60$ & 10 & 22 \\
\hline $61-70$ & 6 & 13 \\
\hline $71-80$ & 2 & 4 \\
\hline Median (range) & $49(22-78)$ & \\
\hline \multicolumn{3}{|l|}{ Race/Ethnicity } \\
\hline White & 12 & 26 \\
\hline Black/African American & 8 & 17 \\
\hline Hispanic & 21 & 46 \\
\hline Asian & 1 & 2 \\
\hline Non/hispanic/Other & 4 & 9 \\
\hline \multicolumn{3}{|l|}{ FIGO Stage at Diagnosis } \\
\hline $\mathrm{Ib} 1$ & 1 & 2 \\
\hline $\mathrm{Ib} 2$ & 7 & 15 \\
\hline IIA & 1 & 2 \\
\hline IIB & 15 & 33 \\
\hline IIIB & 16 & 35 \\
\hline IVA & 6 & 13 \\
\hline \multicolumn{3}{|l|}{ Pathology } \\
\hline Squamous Cervical Carcinoma & 40 & 85 \\
\hline Cervical Adenocarcinoma & 4 & 9 \\
\hline Other & 2 & 4 \\
\hline
\end{tabular}

between involved nodes that did and did not have a CR; weights based on squared residuals were added to reduce the influence of outliers with high leverage. This model controlled for nodal size, nodal type (pelvic or para-aortic), and controlled for the fact that several nodes can come from the same patient (within-patient correlation). A weighted Cox proportional hazard model was constructed to determine if dose is associated with the time it took to achieve a CR. For patient-wise analysis, a model was created which analyzed dose to the lymph nodes on a per patient basis. This model averaged all of the nodal doses per patient and then correlated this average patient dose to patient outcomes. Wilcoxon rank sum tests were used to compare the average initial node size and average nodal dose for patients who had pelvic/PPR, distant recurrence (DR), and total recurrence (TR). A weighted Cox proportional hazard model was constructed to determine if dose was associated with the time until PPR, DR, or TR. Kaplan-Meier analyses were performed to compare the differences between patients with pelvic or pelvic and paranodes in overall survival, distant recurrence-free survival (DRFS), and pelvic/PPR survival (PPRFS), respectively. Kaplan-Meier analyses comparing PPRFS, DRFS, and TR-free survival (TRFS) between patients who had at least one node completely respond and patients who had no nodes completely respond were constructed. Finally, Kaplan-Meier analyses comparing PPRFS, DRFS, and TRFS between patients who had all nodes completely respond and patients who had no nodes completely respond were also constructed.

\section{Results}

The patients' ages ranged from 22 to 78 years with a median of 49 years. The number of patients with stage I, II, III, and IV disease were $8,16,16$, and 6 , respectively. There were 113 pelvic lymph nodes with a median total dose of 55.2 Gy and there were twenty para-aortic nodes with a median dose of 54.4 Gy.

\section{Nodal outcomes}

A generalized estimating equation model looking at all involved lymph nodes showed that even when controlling for node size, node type, and within-patient correlation, increased nodal dose was associated with an increased probability of CR $(P=0.005)$. Figure 1 shows the relationship between dose and probability of study defined CR for pelvic and para-aortic nodes. Table 2 demonstrates this relationship between the probability of CR and a change in external beam dose for nodes with different initial sizes. For a node with an initial size $10 \mathrm{~mm}$ which receives an external beam dose of $60 \mathrm{~Gy}$, the probability of $\mathrm{CR}$ is $65 \%$ while the probability of CR for an identical node which receives 70 Gy is $83 \%$. While there was an association between increased nodal size and decreased probability of $\mathrm{CR}$, this association did not reach statistical significance $(P=0.26)$. A weighted Cox proportional hazard model showed no association between dose and the time it took to achieve a CR, after controlling for nodal size, nodal type, and within-patient correlation $(P=0.797)$.

\section{Patient outcomes}

The median PPRFS, DRFS, and TRFS for the cohort were 20, 9, and 8 months, respectively. For patients who had a CR for all nodes, DRFS and TRFS were 13.8 months and the median PPRFS was not reached. For patient-wise analysis, a Cox 
proportional hazard model showed no statistically significant association between dose and the rate at which patients had PPR, DR, and TR ( $P=0.353,0.818$ and 0.898 , respectively). Wilcoxon rank sum tests were used to compare the average nodal dose for patients with and without recurrences. No statistically significant association was found for PPR $(P=0.263)$, DRs $(P=0.785)$, and TR $(P=1.00)$. Patients with a PPR received 50.0 Gy compares to 51.6 Gy for patients who did not have a PPR. Wilcoxon rank sum tests were used to compare the average initial nodal size for patients with and without recurrences. No statistically significant association was found for PPR $(P=0.368)$, DRs $(P=0.116)$, and TR $(P=0.198)$.

Many Kaplan-Meier analyses were performed for different clinical outcomes. Three Kaplan-Meier analyses were created comparing the median time for PPRFS, DRFS, and TRFS between patients who had at least one node completely respond and patients who had no nodes completely respond. These analyses showed that patients with at least one node completely responded had significantly longer median time for PPRF $(P=0.026)$ [Figure 2a] and TRFS $(P=0.046)$ [Figure 2b]. Patients with at least one node responding also had longer median time for DRFS, but this association was not statistically significant $(P=0.075)$. Three Kaplan-Meier analyses were also constructed comparing the median time for PPRFS, DRFS, and TRFS between patients who had all their nodes completely respond and patients who had none of their nodes completely respond. Median times for PPRF was longer in patients who had all their nodes completely respond $(P<0.05)$ [Figure $2 \mathrm{c}$ ]. Median times for DRFS and TRFS also were greater in patients who had all nodes completely respond, but this difference was not statistically significant ( $P=0.094$ and 0.089 , respectively). Table 3 provides the median recurrence-free survival times and summarizes the results for the six Kaplan-Meier analyses that compared recurrence between patients with different degrees of radiographic CR. Kaplan-Meier analyses were also constructed comparing the patients with only pelvic nodes and patients with pelvic and para-aortic nodes. These analyses found no statically significant difference for PPRFS $(P=0.56)$ or DRFS $(P=0.901)$.

\section{Discussion}

The optimal dose to pelvic and para-aortic lymph nodes is still unclear. There have been several conflicting studies advocating for and against higher doses. Some researchers have opposed increasing the dose to lymph nodes, ${ }^{[2]}$ while other researchers

have been supportive of increasing the dose to lymph nodes in some cases. Rash et al. found an association between increased nodal dose and decreased SUV, ${ }^{[3]}$ and Beriwal et al. hypothesized that a decrease in SUV would improve the negative prognosis associated with lymph node involvement. ${ }^{[6]}$ Wakatsuki et al. suggested that poorly responding nodes may benefit from a boost to at least $58 \mathrm{~Gy}^{[7]}$

The results of our study are essentially in agreement with the studies showing the value of radiation dose. We show that increased dose is associated with an increased probability of nodal CR $(P=0.005)$. For a pelvic node with an initial short axis size $10 \mathrm{~mm}$ which receives an external beam dose of $60 \mathrm{~Gy}$, the probability of $\mathrm{CR}$ is $65 \%$ while the probability of CR for an identical node which receives 70 Gy is $83 \%$. It appears from our study that doses should be close to or $>60 \mathrm{~Gy}$ if technically safe and possible which is our current clinical practice.

Previous studies have shown that nodes that decrease in size after receiving radiation are associated with improved clinical outcome. ${ }^{[7]}$ Our median PPRFS, 20 months, was lower than that of several other studies probably as our median nodal doses were lower and since we included both pelvic and para-aortic nodes in our study. The 2-year disease-free survival for Rash et al. and Beriwal et al. was 50 and $51 \%$, respectively; Wakatsuki et al. had an $82 \%$ pelvic lymph node control rate at 5 years for pelvic node positive patients..$^{[3,6,7]}$ Interestingly, patients who had nodes that completely responded

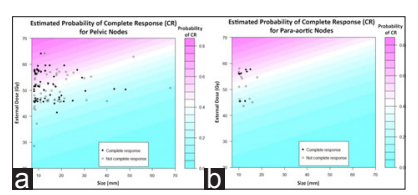

Figure 1: These diagrams illustrate the probability of nodal complete response given the initial size of that node and the external beam nodal dose for pelvic nodes (a) and para-aortic nodes (b)

Table 2: The probability of complete radiographic response for pelvic and para-aortic nodes at different doses

\begin{tabular}{|c|c|c|c|c|c|c|}
\hline \multicolumn{7}{|c|}{ Probability of Complete Radiographic Response for Pelvic Nodes $(95 \%$ CI) } \\
\hline & \multicolumn{6}{|c|}{ Size $(\mathrm{mm})(\%)$} \\
\hline & & 10 & 15 & 20 & 25 & 30 \\
\hline \multirow[t]{4}{*}{ External Dose to Pelvic Nodes (Gy) } & 40 & $21(10-39)$ & $19(9-37)$ & $17(8-35)$ & $15(6-33)$ & $14(5-33)$ \\
\hline & 50 & $42(30-54)$ & $38(28-50)$ & $35(24-48)$ & $32(20-48)$ & $30(16-48)$ \\
\hline & 60 & $65(46-81)$ & $62(43-78)$ & $59(40-76)$ & $56(36-74)$ & $53(31-74)$ \\
\hline & 70 & $83(55-95)$ & $81(53-94)$ & $79(50-94)$ & $77(46-93)$ & $75(42-92)$ \\
\hline \multicolumn{7}{|c|}{ Probability of Complete Radiographic Response for Para-aortic Nodes (95\% CI) } \\
\hline \multirow[t]{4}{*}{ External Dose to Para-aortic Nodes (Gy) } & 40 & $18(7-42)$ & $16(6-39)$ & $15(5-37)$ & $13(4-36)$ & $12(3-35)$ \\
\hline & 50 & $37(17-64)$ & $34(15-61)$ & $31(13-58)$ & $28(11-57)$ & $26(9-56)$ \\
\hline & 60 & $61(28-86)$ & $58(26-84)$ & $54(23-83)$ & $51(20-81)$ & $48(17-80)$ \\
\hline & 70 & $80(38-96)$ & $78(36-96)$ & $76(33-95)$ & $73(29-95)$ & $71(26-94)$ \\
\hline
\end{tabular}


Table 3: Statistical correlation with nodal response and recurrence type

\begin{tabular}{|c|c|c|c|c|c|}
\hline \multicolumn{6}{|c|}{ Kaplan Meier Analyses } \\
\hline \multicolumn{6}{|c|}{ Patients with at Least One Completely Responded Node Versus Patients With No Nodes Completely Responded } \\
\hline \multirow[t]{2}{*}{ Recurrence Type } & \multirow[t]{2}{*}{$P$ value } & \multirow[t]{2}{*}{ Patient Group } & \multirow{2}{*}{$\begin{array}{l}\text { Median Recurrence } \\
\text { Free Survival (Days) }\end{array}$} & \multicolumn{2}{|c|}{ 95\% Confidence Interval } \\
\hline & & & & Lower Bound & Upper Bound \\
\hline \multirow[t]{2}{*}{ Pelvic/Para-aortic Recurrence } & 0.027 & At Least One Node Completely Responded & \# & 280 & * \\
\hline & & No Nodes Completely Responded & 223 & 87 & * \\
\hline \multirow[t]{2}{*}{ Distant Recurrence } & 0.075 & At Least One Node Completely Responded & 364 & 187 & 569 \\
\hline & & No Nodes Completely Responded & 134 & 87 & $*$ \\
\hline \multirow[t]{2}{*}{ Any Recurrence } & 0.046 & At Least One Node Completely Responded & 364 & 187 & 547 \\
\hline & & No Nodes Completely Responded & 134 & 87 & 343 \\
\hline \multicolumn{6}{|c|}{ Patients with All Nodes Completely Responded Versus Patients With No Nodes Completely Responded } \\
\hline \multirow[t]{2}{*}{ Pelvic/Para-aortic Recurrence } & 0.049 & All Node Completely Responded & $\#$ & 190 & $*$ \\
\hline & & No Nodes Completely Responded & 223 & 87 & * \\
\hline \multirow[t]{2}{*}{ Distant Recurrence } & 0.094 & All Node Completely Responded & 420 & 184 & * \\
\hline & & No Nodes Completely Responded & 134 & 87 & * \\
\hline \multirow[t]{2}{*}{ Any Recurrence } & 0.089 & All Node Completely Responded & 420 & 184 & 588 \\
\hline & & No Nodes Completely Responded & 134 & 87 & 343 \\
\hline
\end{tabular}

"\#" indicates that the median survival was not reached. "*” indicates that there was insufficient data to calculate a value

had greater median PPRFS and TRFS compared to patients who did not have any nodes completely respond. Two Kaplan-Meier analyses showed greater PPRFS $(P=0.026)$, and TRFS $(P=0.046)$ in patients who had at least one node completely respond compared to patients who had no nodes completely respond. Furthermore, the Kaplan-Meier analysis looking at patients who had all their nodes completely respond versus patients who had no nodes completely respond also showed that patients with all their nodes completely responded had a longer PPRFS $(P<0.05)$.

Our study demonstrates that increased nodal dose was associated with increased probability of CR. Nodal CR was a positive predictive indicator for improved clinical outcomes. Patients who had nodes that completely responded had statistically significant improvement in median PPRFS. There was also a trend toward improved DRFS in patients who had nodal $\mathrm{CR}$, but this trend failed to reach statistical significance. There were several limitations associated with this study. Not all of our patients received PET/CT at baseline or at disease progression due to limited resources in our public hospital. However, given that LACC is most commonly found in resource-poor countries that lack access to advanced imaging such as PET, our results using CT based nodal measurements are applicable to the majority of situations in those countries. In this study, we chose to include all pelvic nodes $\geq 8 \mathrm{~mm}$ in our analysis per the recommendations of Koh et al. ${ }^{[4]}$ This size cutoff was chosen to increase the sensitivity of detecting involved lymph nodes. A study by Bailey et al. at this institution found that if an $8 \mathrm{~mm}$ cutoff was used, all PET avid nodes were identified, but if a $10 \mathrm{~mm}$ cutoff was used, 13 of 58 patients (22\% of patients) had metabolically active nodes that would not have been identified. ${ }^{[8]}$ However, Einstein et al. recommend a larger cutoff be used due to the fact that nodes from $8 \mathrm{~mm}$ to $10 \mathrm{~mm}$ could be normal. ${ }^{[9]}$ Using a lower size cutoff decreases the specificity for metastatic nodes. Because our patients did not have access to PET scans, we chose the lower $8 \mathrm{~mm}$ cutoff.

Another limitation is the lack of follow-up PET scan posttreatment and its replacement with serial CT scans. PET

South Asian Journal of Cancer • Volume 6 •Issue 2 • April-June 2017 scan is an extremely expensive test and is not available at our public hospital in follow-up. One criticism of this approach is the moderate sensitivity of the CT for lymph node involvement $(31 \%){ }^{[10]}$ However, we were following decreases in lymph node size which is measured accurately by CE-CT. The issue of repeated radiation exposure with CT scan is also brought up but PET scan also involves some radiation exposure.

Only 46 patients were included in this study. An increased sample size would increase the power of our study and increased the probability of detecting clinically significant factors. Furthermore, follow-up time varied due to some patients being treated at an earlier date than others. An increased follow-up time would improve the validity of the study. Finally, as a nonrandomized retrospective study, it is subject to data collection biases, interpretation biases and can only show association not causation. Larger prospective clinical trials are needed to definitively determine if increased nodal dose can decrease the occurrence of pelvic and para-aortic recurrence.

\section{Acknowledgments}

We would like to acknowledge the support of the University of Texas Southwestern Radiation Oncology and Radiology Departments and the University of Texas Southwestern Summer Medical Student Research Program.

\section{Financial support and sponsorship}

Nil.

\section{Conflicts of interest}

There are no conflicts of interest.

\section{References}

1. Toita T, Nakano M, Higashi M, Sakumoto K, Kanazawa K. Prognostic value of cervical size and pelvic lymph node status assessed by computed tomography for patients with uterine cervical cancer treated by radical radiation therapy. Int J Radiat Oncol Biol Phys 1995;33:843-9.

2. Grigsby PW, Singh AK, Siegel BA, Dehdashti F, Rader J, Zoberi I. Lymph node control in cervical cancer. Int J Radiat Oncol Biol Phys 2004;59:706-12.

3. Rash DL, Lee YC, Kashefi A, Durbin-Johnson B, Mathai M, Valicenti R, et al. Clinical response of pelvic and para-aortic lymphadenopathy to a radiation boost in the definitive management of locally advanced cervical cancer. Int J Radiat Oncol Biol Phys 2013;87:317-22. 
4. Koh DM, Hughes M, Husband JE. Cross-sectional imaging of nodal metastases in the abdomen and pelvis. Abdom Imaging 2006;31:632-43.

5. Griffeth LK. Use of PET/CT scanning in cancer patients: Technical and practical considerations. Proc (Bayl Univ Med Cent) 2005; 18:321-30.

6. Beriwal S, Gan GN, Heron DE, Selvaraj RN, Kim H, Lalonde R, et al. Early clinical outcome with concurrent chemotherapy and extended-field, intensity-modulated radiotherapy for cervical cancer. Int J Radiat Oncol Biol Phys 2007;68: 166-71.

7. Wakatsuki M, Ohno T, Kato S, Ando K, Noda SE, Kiyohara H, et al. Impact of boost irradiation on pelvic lymph node control in patients with cervical cancer. J Radiat Res 2014;55:139-45.

8. Bailey A, Hwang L, Xi Y, McKeever M, Albuquerque K. CT Mapping of
Metastatic Nodal Disease in Patients with Advanced Cervical Cancer in an Indigent US Population: Implications for Resource Utilization and Conformal Radiation (CRT) Planning, in Radiological Society of North America 2014 Scientific Assembly and Annual Meeting. Chicago IL; 2014.

9. Einstein DM, Singer AA, Chilcote WA, Desai RK. Abdominal lymphadenopathy: Spectrum of CT findings. Radiographics 1991;11:457-72.

10. Mitchell DG, Snyder B, Coakley F, Reinhold C, Thomas G, Amendola MA, et al. Early invasive cervical cancer: MRI and CT predictors of lymphatic metastases in the ACRIN 6651/GOG 183 intergroup study. Gynecol Oncol 2009; 112:95-103. 